\title{
Audit of head injury management in Accident and Emergency at two hospitals: implications for NICE CT guidelines Lucy Miller ${ }^{1}$, Ruth M Kent*2 and Alan Tennant ${ }^{2}$
}

Address: ${ }^{1}$ The Medical School, The University of Leeds. Worsley Building, Clarendon Way, Leeds UK. LS2 9JT and ${ }^{2}$ Academic Unit of Musculoskeletal and Rehabilitation Medicine, The University of Leeds, 36 Clarendon Road, University of Leeds, Leeds, UK. LS2 9NZ

Email: Lucy Miller - ugm0I2m@leeds.ac.uk; Ruth M Kent* - r.m.kent@leeds.ac.uk; Alan Tennant - a.tennant@leeds.ac.uk

* Corresponding author

Published: 03 May 2004

BMC Health Services Research 2004, 4:7
Received: 12 November 2003

Accepted: 03 May 2004

This article is available from: http://www.biomedcentral.com/I472-6963/4/7

(C) 2004 Miller et al; licensee BioMed Central Ltd. This is an Open Access article: verbatim copying and redistribution of this article are permitted in all media for any purpose, provided this notice is preserved along with the article's original URL.

\begin{abstract}
Background: The National Institute for Clinical Excellence (NICE) has produced guidelines on the early management of head injury. This study audits the process of the management of patients with head injury presenting at Accident and Emergency (A\&E) departments and examines the impact upon resources of introducing NICE guidelines for eligibility of a CT scan.

Methods: A retrospective audit of consecutive patients of any age, presenting at A\&E with a complaint of head injury during one month in two northern District General Hospitals forming part of a single NHS Trust.

Results: 419 patients presented with a median age of $I 5.5$ years, and $61 \%$ were male. $58 \%$ had a Glasgow Coma Score (GCS) recorded and 33 (8\%) were admitted. Only four of the ten indicators for a CT scan were routinely assessed, but data were complete for only one (age), and largely absent for another (vomiting). Using just three (incomplete) indicators showed a likely 4 fold increase in the need for a CT scan.

Conclusions: The majority of patients who present with a head injury to Accident and Emergency departments are discharged home. Current assessment processes and associated data collection routines do not provide the information necessary to implement NICE guidelines for CT brain scans. The development of such clinical audit systems in a busy A\&E department is likely to require considerable investment in technology and/or staff. The resource implications for radiology are likely to be substantial.
\end{abstract}

\section{Background}

The National Institute for Clinical Excellence (NICE) has produced guidelines entitled 'Head Injury in Infants, Children and Adults: Triage, Assessment, Investigation and Early Management' [1]. In the document, guidelines are presented which recommend good practice in Accident and Emergency (A\&E) assessment and, specifically, what are the indicators for a Computerised Tomography (CT) scan (Figure 1). CT scanning is almost 100\% sensitive and specific for the identification of intracranial complications. In comparison, a skull $\mathrm{x}$-ray is $38 \%$ sensitive and 95\% specific for skull fracture [2]. One reason for this poor sensitivity is the unreliability of the interpretation of the $\mathrm{x}$-ray by under-pressure A\&E clinicians.

The identification of intracranial complications is important as such problems are not rare. In one study, $47 \%$ of patients admitted to hospital with a head injury were 
a)

- The priority is stabilisation of airways, breathing and circulation.

- Patients with impaired consciousness (GCS <15) should be assessed immediately by a trained member of staff (e.g. triage nurse) in order to establish whether they are high or low risk for clinically important brain injury using the Canadian rules.

- Patients should be assessed by an A\&E clinician to fully establish the need for CT imaging of the head, again based on the Canadian rules. Patients found to be high risk on triage for clinically important brain injury should be assessed more urgently than those at low risk.

- Patients should not receive systemic analgesia until fully assessed so that an accurate measure of consciousness and other neurological signs can be made.

- Care professionals should use a standard head injury proforma in their documentation when assessing and observing head injured patients. A separate proforma should be used for those under 16 years of age. b)

- GCS less than 13 at any time since injury

- GCS 13 or 14 two hours post injury

- Suspected open or depressed skull fracture

- Any sign of basal skull fracture (haemotympanum, 'panda' eyes, cerebrospinal fluid otorrhoea, Battle's sign)

- Post-traumatic seizure

- Focal neurological deficit

- More than one episode of vomiting

- Amnesia of more than 30 minutes before the event

- Coagulopathy (history of bleeding, clotting disorder, current warfarin treatment)

- Dangerous mechanism of injury (pedestrian struck by a vehicle, occupant ejected from a vehicle, fall from a height of greater than three feet or five stairs)

- Age greater or equal to 65 years where evidence of loss of consciousness or amnesia experienced

Source: National Institute for Clinical Excellence. Triage, assessment. Investigation and early management of head injury in infants, children and adults. Clinical Guidelines 4. NICE, 2003.

Figure I

NICE guidelines for a) early management of head injury and for b) the need for a CT scan.

found to have a disability one year post-injury [3]. The problem of identifying high-risk patients was first tackled in 1984 with the introduction of UK-wide guidelines [4]. They were based mainly on the level of consciousness to establish whether head injured patients were at high or low risk of intracranial complications. Skull x-ray was the main investigation as skull fracture was considered to be a risk factor for intracranial complications. The guidelines have since been modified to include CT scanning, using the Glasgow Coma Scale (GCS) and presence of a skull fracture as well as various other clinical factors [5].

Given the incidence of head injury in the United Kingdom [3] the proposed indicators for CT in the NICE document have a number of practice and resource implications both with respect to the information needs which underpin the indicators, as well as in the potential demand for the CT scan. Current practice would appear to reserve CT 
scanning for those deemed to be at high risk of intracranial injury, whereas the NICE guidelines emphasise the importance of CT scanning as part of a screening process above a threshold of risk.

\section{Methods}

A retrospective clinical audit was undertaken at two northern District General Hospitals, which form a single NHS Trust. These hospitals are of medium size, with a joint annual Accident and Emergency new attendance of 112,000 . The audit set out to determine:

- What happens to head injured patients from presentation at A\&E to discharge?

- What information is collected from presentation to discharge?

- What are the implications of CT guidelines for information collection and referral rates to radiography?

Information was thus collected on all patients presenting at the two A\&E departments for the calendar month of April 2003 with an initial complaint of head injury. Patient details were extracted from the central computer system using search criteria of attendance at A\&E in April 2003 as well as the initial complaint of head injury. The definition was according to local protocols and coding, and those with primarily facial injuries are excluded. Data was obtained for each patient with respect to their name, attendance date, hospital number, and date of birth, sex, arrival time, departure time, destination, disposal, and initial complaint.

Additional data was collected retrospectively from the A\&E records on which information was recorded contemporaneously at presentation of the head injured patients. This included the mode of arrival at hospital, Glasgow Coma (GCS) Score [6] at different stages, vomiting (if more than twice children are kept in), memory of events, any investigations or treatment required, destination after leaving A\&E, whether the GP was contacted, and diagnostic-related coding.

The proposed audit was discussed with the chair of the local ethics committee who confirmed the audit status of the work.

\section{Results}

\section{Pathways of care}

A total of 419 patients presented at the two A\&E departments during April 2003 with the initial complaint of head injury. Median age was 15.5 years (IQR 4-35) and $61 \%$ were male. Just over one-quarter $(26 \%)$ were brought by ambulance. When data for the two hospitals was compared, there was no significant difference between hospitals in the ages of those presenting $(t=-$ $1.397 ; \mathrm{p}=0.163$ ), in their gender (Chi-Square $=0.204 ; \mathrm{p}$ $=0.652)$, or in GCS at presentation $(t=-0.102 ; p=0.919)$. Given this, the data was combined for further analysis.

Initially patients are triaged, and subsequently admitted or discharged after review. Figure 2 shows a summary of what happened to the patients according to their GCS on presentation. Of the 419 patients presenting, only 244 (58\%) had their GCS recorded. Of those where GCS was not recorded, 26 (15\%) did not wait to be further assessed by a doctor after triage. Eleven of those remaining in the department $(7 \%)$ were admitted to hospital. Of the 244 patients (58\% of the total) whose GCS was recorded, 9 (4\%) did not wait for review. Of those who waited, 11 (5\%) had a GCS of less than 15 on presentation, 7 of whom $(64 \%)$ were admitted, with the remainder discharged to their previous place of residence. Of those who waited in A\&E and had a GCS of 15, $15(7 \%)$ required admission.

Of the 33 head injured patients admitted 14 (42\%) were children under 16 years of age, all of whom were discharged with 48 hours. 13 patients aged $16-74$ years were admitted and 10 of these (77\%) stayed for less than 48 hours. Of these three who stayed longer, two were transferred to a neurosurgery department following a CT scan, one of whom was subsequently discharged home with definite neuropsychological sequelae. This patient was subsequently referred back to rehabilitation. Of the elderly group, only $17 \%$ were discharged within 48 hours. Two patients subsequently died in hospital and the remainder were discharged home. Cause of death was not recorded as part of the audit.

\section{Information recorded}

Less than three-in-five (58\%) had a GCS recorded. The number of times GCS was recorded within the A\&E department was found to be significantly higher in the population who were admitted, as might be expected under these circumstances (mean 1.03 in discharged population, compared to admitted population, mean 2.73) (t $=-2.936 ; \mathrm{p}=<0.01)$. Whether the patient reported any amnesia (as either present or absent) was recorded in $7 \%$ of cases, and this (recorded or not) was found to have no association with admission (Chi-Square $=1.998 ; \mathrm{p}=$ 0.157).

Several procedures and interventions were recorded. Assessment of the pupils was common, with 290 patients $(70 \%)$ having their reactivity recorded and $58 \%$ having their GCS recorded. Considering radiological investigations, 142 (34\%) of the presenting 419 patients had a skull $\mathrm{x}$-ray recorded with $27 \%$ of children under 16 


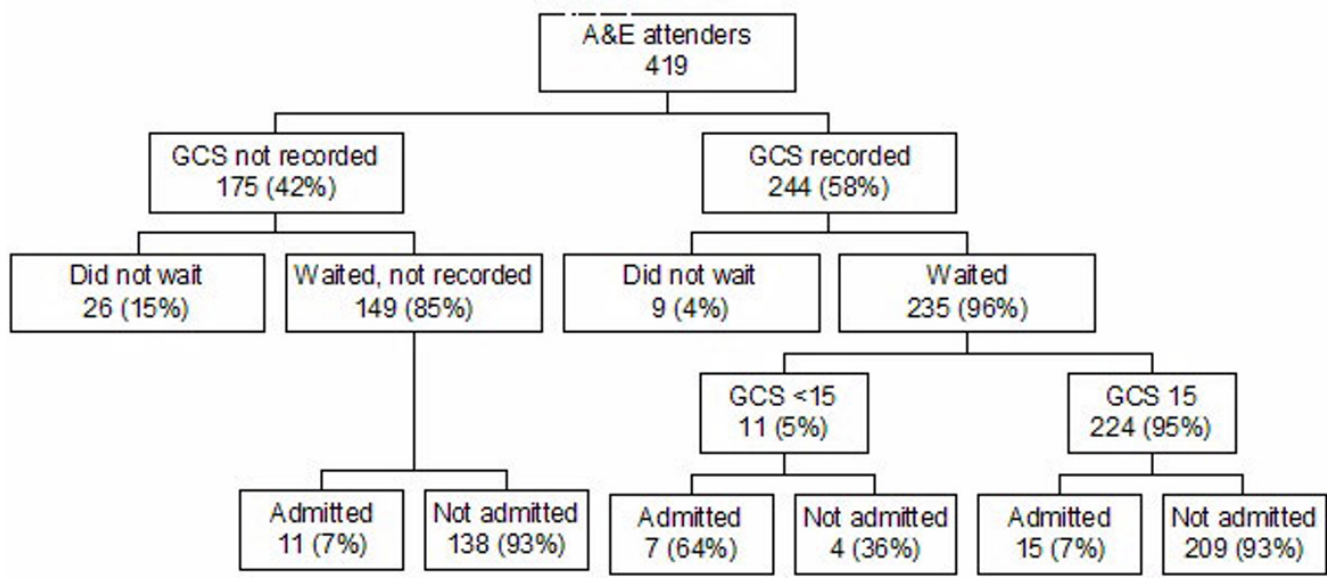

Figure 2

Care pathways for presentation of head injury at two District General Hospitals.

receiving a skull $\mathrm{x}$-ray, $38 \%$ of those aged $16-74$ and $59 \%$ of the 75 and over. In comparison, 6 out of 419 patients had a CT scan of their head recorded, which is equivalent to $1 \%$. Four of these were performed when the GCS was less than 13, one when the GCS was 15 , and one when GCS was not recorded.

Of the patients who were discharged home from $A \& E$, $64 \%$ were recorded as being given a head injury advice card which provides information about the possible complications of head injury and symptoms to look out for, indicating that the patient should return to A\&E. A 24hour telephone number was also available for each hospital. Over four in five (85\%) of patients' GPs were contacted after their discharge from A\&E with specific clinical details of the head injury; all others received a routine computer generated notification of attendance. It is not clear why this was so in certain cases. In this case a significant difference was found between hospitals (Chi-Square $=11.827 ; \mathrm{p}=<0.001)$.

\section{Guidelines for indications for a CT scan of the head}

Of the ten indicators listed in the NICE guidelines as indicative of a CT scan, only four were routinely collected in these two hospitals, and only one, age, and had complete data. As we do not have reliable recording of amnesia we have used three indicators for the calculation of increased radiology workload.

These indicators include 1) the number of people with a GCS of less than 13 on presentation; 2) the number of people aged 65 and over and 3) those given an x-ray and subsequently admitted (Admission following skull x-ray is used as a proxy indicator for concern about findings of the x-ray, or suspicion about serious mechanism of injury). We then modified indicator 2 , which accords with the Canadian CT guidelines [7] upon which the NICE guidelines were based, and constrained those aged over 65 to have an additional indicator present. Thus we can give crude (lowest) estimates for those who are likely to conform to Canadian guidelines, through to those likely to be eligible under the NICE guidelines, which add conditions to those aged over 65 years (Figure 1).

From this we are able to estimate that 55 patients would have met the requirements for a CT scan under the Canadian guidelines, through to 26 eligible for a CT scan under the NICE guidelines, 5 of whom were over 65 . The audit shows that all six people who were scanned met the NICE criteria.

These data suggest that four times as many CT scans of the head will be required in each hospital under NICE guidelines, as are presently undertaken, and up to nine times as many under the less constrained Canadian guidelines. As this estimate is based on only three of the ten indicators, two of which were incomplete, this range (of 4-9) should be considered as the minimum increase in the number of scans required with the Canadian based estimate as the upper bound of demand. 


\section{Discussion}

Head injury is a common condition presenting to Accident and Emergency departments. The rate audited in this report represents an annual presentational incidence of 1520 per 100,000 people for the locality. The extrapolated annual hospitalised incidence rate is 126 per 100,000, much lower than other published data [8]. However, four factors may influence this. Firstly extrapolating from one month may lead to bias in estimates, either under- or over estimating incidence presented. Secondly, incidence is known to vary considerably by locality, predicated on demographic and socio-economic factors [8] and, thirdly, some of the most severe patients may be triaged by the ambulance service directly to the regional neurosurgical centre. Nevertheless, the latter will be relatively few in number. Finally, the changing management of people presenting, with greater emphasis on sending people home with information cards, rather than admitting for observation, possibly as a result of a reduction in short stay "observation" beds over the last decade. This may be having a general effect in reducing admission rates and thus reducing the viability of historical compatibility.

It is likely that the guidelines introduced in 1984 and since modified have contributed much to the increase in the number of CT scans requested and performed on head injured patients in the UK $[1,8]$. This has been made possible by the greater availability of 24 hour CT scanning in the UK. Nevertheless, current practice in the UK CT scanning is reserved for patients considered to be at high risk of intracranial complications, whereas in the USA, CT scanning is performed in $75-100 \%$ of patients with a normal GCS and loss of consciousness [9]. The results of this audit show that only a small number of CT scans were done, but should the NICE guidelines be implemented then the number would be increased at least four-fold in these two hospitals.

Another important finding of the audit was the incomplete recording of information within the A\&E departments, despite both using the same protocol. Those patients who decide to go home before any information can be recorded will contribute to this loss of information. The absence of recorded information does not of course imply the absence of the action, but in a busy A\&E some mechanism must be introduced to facilitate accurate recording of activities. One solution would be to introduce a standardised proforma for head injured patients in A\&E [1]. This could have a checklist of tests, procedures, and interventions, which may be required for management, including collecting the relevant indicators for a CT scan. A reminder to ask about amnesia, including distinction between ante grade and retrograde amnesia may be prompted for, as required by the NICE guidelines. However, in the two hospitals audited, this would require a radical overhaul of existing documentation, which currently contains only a designated area for recording GCS and pupil reactivity and even these, as we have seen, are not always completed.

A number of patients do not wait to be seen in the A\&E department after initial triage, some of whom do not have their GCS recorded, yet if the NICE guidelines are going to be followed then a number of these people may require scanning. This raises potential medico-legal issues. Another important point is that admission decisions appear to be made on other criteria apart, or additional to initial GCS, as equal proportions of those where the GCS was recorded or not were admitted. Thus the potential impact of the implementation on the NICE guidelines on admission criteria and hence use of resources will also need to be considered. Likewise, some thought will need to the impact on the admission of children with head injuries. Whereas just two children were given a scan during the audit period, our results would imply that 10 would be eligible, $80 \%$ of whom had been given a skull $\mathrm{x}$ ray.

The audit has a number of limitations. There were some problems in case ascertainment with finding the $\mathrm{A} \& \mathrm{E}$ cards. In both A\&E departments some cards could not be found. The study relied on the accuracy of the identification and recording of head injury by the A \& E staff. They were filed in alphabetical order by hand, making it easy for cards to be filed in the wrong place due to human error. In one hospital all the casualty cards were filed together in drawers, whereas at another cards for children under the age of 16 years were filed separately. There is also no way of telling whether or not these two hospitals are in any way representative of District General Hospital A\&E departments in general viz. staffing, skill levels and local protocols, all of which may be highly variable throughout the UK. The variables chosen to record were those most frequently recorded at the time of the audit, to take a snapshot of the impact of the guidelines. A more complete picture of the CT scanning and admission policy may have been given if we had screened for those (albeit more rare) factors such as a dangerous mechanism of injury or those with a suspected coagulopathy. Given accurate recording of such factors, a future audit would look at their incidence over a longer time frame.

The audit process, from presentation at $\mathrm{A} \& \mathrm{E}$ to discharge at the two hospitals, has also shown that what practitioners believe happens in their department is not borne out by data, most strikingly the recording of the GCS. The senior consultant in A\&E for the trust remarked that the disparity between what they thought had been done and what had actually been recorded, but commented that the increased requirements for CT scanning was not surpris- 
ing. Furthermore, if careful documentation of CT indicators is required for all patients presenting with head injury, this must have a resource implication for the numbers of staff on duty, or the wait times, or both. It is possible that medical informatics systems may be able to make a substantial contribution to the information processing and management of those presenting with a head injury, relieving hard pressed staff of some of the burden of data recording and ensuring (perhaps through touch screen technology) that relevant data is recorded. However, once again there are developmental and equipment resource implications in seeking such a solution.

Although senior clinicians in Accident and Emergency medicine are aware of the NICE guidelines, it is possible that in many places it has not been integrated into medical practice. The reasons for this may be lack of knowledge amongst junior staff, which could be addressed by the adoption of protocols for recording information or, perhaps at the present time, the availability of radiology services, particularly out of hours.

Future developments to facilitate better management of those presenting with a minor head injury may also include the identification of patients at risk of longer-term sequelae $[10,11]$. Such a process may utilise the power of informatics systems using recent developments in measurement technologies [12] to minimise the burden of assessment for both staff and patients.

\section{Conclusion}

Most people presenting to the A\&E departments with a head injury in the two hospitals were discharged straight home from the department. The audit showed that of those subsequently admitted, $76 \%$ were in hospital for less than 48 hours. Data was found to be incomplete around the GCS, vomiting, amnesia and the length of any loss of consciousness, all being indicators in the NICE guidelines for a CT scan of the head. A considerable investment in the recording of information will be required if guidelines such as those recommended by NICE are to be implemented effectively. The current evidence is that if the NICE CT guidelines were implemented, a minimum four-fold increase in scans would be required, and all that implies for radiology staffing. A prospective audit, including all the NICE guidelines, is currently underway in the two hospitals.

\section{Competing interests}

None declared.

\section{Authors' contributions}

RK and AT designed the study. LM carried out the audit under the supervision of RK. LM and AT performed the statistical analysis. All authors read and approved the final manuscript.

\section{Acknowledgements}

We would like to thank the consultants and other staff at the two hospitals who facilitated this audit and particularly Mr Michael J Playforth for his helpful comments on a draft of this paper.

\section{References}

I. National Institute for Clinical Excellence: Triage, assessment. Investigation and early management of head injury in infants, children and adults. Clinical Guidelines 4. NICE 2003.

2. Hofman PA, Nelemans P, Kemerink GJ, Wilmink JT: Value of radiological diagnosis of skull fracture in the management of mild head injury: meta-analysis. J Neurol Neurosurg Psychiatry 2000, 68:416-22

3. Thornhill S, Teasdale GM, Murray GD, McEwen J, Roy CW, Penny KI: Disability in young people and adults one year after head injury: prospective cohort study. BMJ 2000, 320:I63|-5.

4. Briggs $M$, Clarke $P$, Crockard A, Jennett B: Guidelines for initial management after head injury in adults. Suggestions from a group of neurosurgeons. BM] 1984, 223:49-52.

5. Scottish Intercollegiate Guidelines Network: Early management of patients with head injury. SIGN 2000.

6. Teasdale G, Jennett B: Assessment of coma and impaired consciousness. A practical scale. Lancet 1974, 2:81-83.

7. Stiell IG, Wells GA, Vandemheen K, Clement C, Lesiuk H, Laupacis $A$, McKnight RD, Verbeek R, Brison R, Cass D, Eisenhauer MA, Greenberg GH, Worthington J: The Canadian CT head rule for patients with minor injury. The Lancet 200I, 357:| 39|-I396.

8. Tennant A: Epidemiology of head injury. in Traumatic Brain Injury Rehabilitation: Services, treatments and outcomes Edited by: Chamberlain MA, Neumann VC, Tennant A. London:Chapman \& Hall; 1995: I 2-24.

9. Livingston DH, Lavery RF, Passannante MR, Skurnick JH, Baker S, Fabien TC, et al:: Emergency department discharge of patients with a negative cranial computed tomography scan after minimal head injury. Ann Surg 2000, 232:126-32.

10. King NS: Emotional, neuropsychological, and organic factors: their use in the prediction of persisting post concussion symptoms after moderate and mild head injuries. I Neurol Neurosurg Psychiatry 1996, 6 I:75-8I.

II. Bazarian JJ, Wong T, Harris M, Leahey N, Mookerjee S, Dombovy M: Epidemiology and predictors of post-concussive syndrome after minor head injury in an emergency population. Brain Injury 1999, 13:173-189.

12. Ware JE Jr, Bjorner JB, Kosinski M: Practical implications of Item Response Theory and Computer Adaptive Testing: A brief summary of ongoing studies of widely used headache impact scales. Med Care 2000, 38(9 Supple II):73-82.

\section{Pre-publication history}

The pre-publication history for this paper can be accessed here:

http://www.biomedcentral.com/1472-6963/4/7/prepub 